\title{
PERFIL NUTRICIONAL DE PRÉ-ESCOLARES E ESCOLARES RESIDENTES EM PALMEIRAS DO JAVARI, AM.
}

\author{
Yolanda Rebelo da ROCHA ${ }^{1}$, Lucia Kiyoko Ozaki YUYAMA ${ }^{1}$, Osvaldo \\ Pinheiro do NASCIMENTO ${ }^{1}$
}

RESUMO - Avaliou-se o perfil nutricional de 26 crianças em idade pré-escolar (2-5 anos) e 56 crianças em idade escolar (6-14 anos), residentes em Palmeiras do Javari, AM. Na caracterização do estado nutricional da população estudada, utilizou-se os parâmetros antropométricos, inquérito alimentar recordatório de 24 horas em conjunto com o da pesagem direta dos alimentos, determinação da hemoglobina e o parasitológico. Os resultados indicaram que dos 26 pré-escolares estudados, $11,5 \%$ apresentavam desnutrição de grau I. Em relação aos escolares $41 \%$ e $9 \%$ apresentaram, respectivamente, desnutrição de grau I e II de acordo com a classificação de GOMEZ. Em relação à alimentação oferecida pela escola, os dados são sugestivos de uma suficiência na ingestão de proteína de origem animal e vitamina $\mathrm{C}$ pelos pré-escolares em quase $100 \%$, e uma deficiência de $49 \%$ e $69 \%$, respectivamente, para vitamina A e ferro. Quanto aos escolares, houve uma deficiência na ingestão de calorias de $65 \%$, Vitamina A $76 \%$, B1 $65 \%$, niacina $60 \%$ e ferro $75 \%$, de acordo com as recomendações diárias (NASNRC 1980). Verificou-se que mais de $50 \%$ das crianças estudadas eram anêmicas, o que nos leva a situar a anemia, como uma das deficiências nutricionais, associadas a alta prevalência de parasitoses intestinais.

Palavras chave: Perfil nutricional, Alimentação, Pré-escolar e Escolar, Antropometria.

Nutritional profile of pre-school children and other school children, residents in Palmeira do Javari, AM

ABSTRACT - The nutritional profile of 26 pre-school children from the age of 2 to 5 years old, and 56 other school children aged from 6 to 14 years old, residents in Palmeira do JavariAm, were evaluated. The characterization of the nutritional state of the population studied included, anthropometric measurements, food enquiry, using the 24 hours recall method in conjunction with the weight of foods, and determination of hemoglobin and parasitological exams. The results of these evaluations showed that of the 26 pre-school children studied, $11.5 \%$ had first degree malnutrition. This compared with the other students, of which $41 \%$ and $9 \%$ presented, respectively, 1 st and 2 st degree malnutrition according to the classification of GOMEZ. In relation to the food offered by the school, the data suggests almost $100 \%$ sufficiency in the ingestion of animal protein and vitamin $\mathrm{C}$ for the pre-school children and a deficiency of $49 \%$ and $69 \%$, respectively, for vitamin $\mathrm{A}$ and iron. The other students, however, showed a deficiency in the calorie intake of $65 \%$,vitamin A $76 \%$, B1 $65 \%$, niacine $60 \%$ and iron $25 \%$, according to the daily recommended intakes (NAS-NRC 1980). It was noted that more than $50 \%$ of the children studied were anemic and it was deduced that anemia comes with nutritional deficiency in association with the rate of intestinal parasites.

Key words: Nutritional profile, Alimentation, Pre-school children and school school children, Antropometrie.

1 Instituto Nacional de Pesquisas da Amazônia-INPA, Coordenação de Pesquisas em Ciências da Saúde, Laboratório de Nutrição, Caixa Postal 478, 69011-970 - Manaus, AM.

ACTA AMAZONICA 23(1): 9-14. 1993. 


\section{INTRODUÇÃO}

A avaliação do estado nutricional de populações é de grande importância na caracterização epidemiológica e sócio-econômica da região. Por representarem um grupo biológicamente vulnerável, as crianças em idade pré-escolar, têm se constituido na principal preocupação de especialistas de saúde pública (VENTAKACHALAN, 1976). É uma faixa etária importante devido ao processo de maturação biológica onde $o$ alimento tem um papel fundamental no seu desenvolvimento psico-somático (ORNELLAS, 1983). É um período da infância em que se encontra uma elevada incidência de desnutrição protéico calórica (KELLER et al., 1976), concorrendo para o atraso do crescimento e desenvolvimento. Os estudos desenvolvidos no estado do Amazonas e região revelam entre si, uma certa semelhança em termos de prevalência de desnutrição, sendo também freqüentes as deficiências vitamínicas como B1, $B 2$, niacina, $A$ e de alguns minerais como cálcio, ferro e zinco (GIUGLIANO \& SHRIMPTON, 1977; HARTMAN \& JORGE JOÃO, 1978; SHRIMPTON \& GIUGLIANO, 1979; GIUGLIANO et al., 1981; 1984; Simpósio Brasileiro de Alimentação e Nutrição, 1984).

As crianças em idade escolar embora normalmente não apresentem sérias enfermidades e mortalidade por sub-nutrị̧ão (JELLIFFE, 1966) são também alvo de nosso estudo, tendo em vista, que agravos nutricionais precoces e repetidos deixam seqüelas nas fases críticas de crescimento e desenvolvimento das crianças.

Considerando que os processos fisiológicos associados à desnutrição envolvem alterações de peso e estatura; o presente estudo teve como objetivo avaliar o estado nutricional de préescolares e escolares residentes na área urbana de Palmeiras do Javari.

\section{MATERIAIS E MÉTODOS}

O estudo foi realizado em uma escola com capacidade para 100 crianças em idade pré-escolar e escolar, em Palmeira do Javari situada à margem direita do rio Javari, fronteira com o Peru, estado do Amazonas. O grupo amostral foi constituído de 26 crianças em idade pré-escolar ( 2 a 5 anos) de ambos os sexos, sendo 20 (77\%) do sexo feminino e 6 (23\%) do sexo masculino, e 56 crianças em idade escolar (6 a 14 anos), com $35(62.5 \%)$ do sexo feminino e $21(37.5 \%)$ do sexo masculino, residentes na área urbana de Palmeira do Javari,que freqüentavam a escola. $\mathrm{O}$ estado nutricional do grupo estudado foi avaliado através dos parâmetros antropométricos, considerando as medidas de peso e altura segundo as normas de JELLIFFE (1966). Utilizouse também o inquérito alimentar recordatório de 24 horas em conjunto com o da pesagem direta dos alimentos oferecidos pela escola. $\mathrm{Na}$ avaliação do estado nutricional de pré-escolares e escolares, os dados antropométricos foram avaliados segundo o sistema de classificação de GOMEZ (1956), e os 
indicadores nutricionais com base nos padrões do NCHS (NELSON, 1979).

Para o cálculo de ingestão de calorias e nutrientes utilizou-se a tabela de composição de alimentos, (AZOUBEL et al., 1982), e (FIBGE, 1977). As necessidades de calorias e nutrientes, seguiu-se as recomendações da National Academy of Sciences e National Research Council (NASNRC). O exame parasitológico foi realizado pelo método quantitativo e qualitativo de exame de fezes e FAUST et al. (1939).

Para a caracterização da anemia utilizou-se a técnica de determinação de hemoglobina pelo método de CIANOMETAHEMOGLOBINA. (OMS, 1968).

\section{RESULTADOS E DISCUSSÃO}

$\mathrm{Na}$ caracterização do estado nutricional do grupo de pré-escolares, verificou-se uma incidência relativamente baixa de desnutrição uma vez que das 26 crianças, $88,5 \%$ eram eutófricas e $11,5 \%$ apresentaram desnutrição de grau I (Tab. 1). O mesmo não ocorreu com o grupo de escolares que apresentaram uma incidência de desnutrição de grau I e II de $50 \%$ (Tab. 1). Não houve registro de desnutrição de grau III. De uma certa forma esses resultados obtidos quando confrontados com outros estudados no estado do Amazonas, revelaram uma certa semelhança em termos de desnutrição (GIUGLIANO \& SHRIMPTON, 1977; GIUGLIANO et al., 1978).
Tabela 1. Avaliação do estado nutricional através de GOMEZ, de pré-escolares e escolares, em Palmeira do Javari-Am.

\begin{tabular}{|c|c|c|c|c|}
\hline \multirow{2}{*}{$\begin{array}{l}\text { Graus de } \\
\text { Gomez }\end{array}$} & \multicolumn{2}{|c|}{ Pré-escolares } & \multicolumn{2}{|c|}{ Escolares } \\
\hline & $\mathbf{N}^{\circ}$ & $\%$ & $\mathbf{N}^{\circ}$ & $\%$ \\
\hline Normal & $\begin{array}{r}23 \\
3\end{array}$ & $\begin{array}{l}88.5 \\
11.5\end{array}$ & $\begin{array}{l}30 \\
21\end{array}$ & $\begin{array}{l}53.5 \\
37.5\end{array}$ \\
\hline II & - & - & 5 & 9.0 \\
\hline III & - & - & - & - \\
\hline Total & 26 & 100 & 56 & 100 \\
\hline
\end{tabular}

A alimentação básica oferecida pela escola era composta de três refeições, ou seja: desjejum, almoço e lanche, com uma contribuição calórica por refeição de $20,5 \%$; $59 \%$ e $20 \%$ respectivamente. As preparações mais freqüentes foram: - Mingau com leite, neston ou farinha láctea. - Leite com café ou nescau e pão-- Carne na forma de bife ou guisado, peixe frito ou ensopado, tendo como guarnição batata ou banana pacovão frita. - Arroz, macarrão e de sobremesa uma fruta ou um doce. A composição média de calorias e nutrientes da alimentação oferecida aos pré-escolares pela merenda escolar encontra-se na Tabela 2. Analisando os valores médios encontrados para a ingestão de nutrientes e calorias em função do

Tabela 2. Média do consumo da dieta de pré-escolares oferecida na escola.

\begin{tabular}{lr}
\hline $\begin{array}{l}\text { Composiçāo da dieta básica } \\
\text { oferecida na Escola. }\end{array}$ & Quantidade \\
\hline Calorias & $863.00 \mathrm{Cal}$ \\
Proteína (origem animal). & $25.15 \mathrm{~g}$ \\
Glicídio & $127.83 \mathrm{~g}$ \\
Lipídio & $27.95 \mathrm{~g}$ \\
Fibra & $1.04 \mathrm{~g}$ \\
Cálcio & $364.30 \mathrm{mg}$ \\
Fósforo & $526.22 \mathrm{mg}$ \\
Ferro & $3.64 \mathrm{mg}$ \\
Sódio & $555.26 \mathrm{mg}$ \\
Vit. A & $138.92 \mathrm{mg}$ \\
Vit. B1 & $0.35 \mathrm{mg}$ \\
Vit. B2 & $0.76 \mathrm{mg}$ \\
Niacina & $6.48 \mathrm{mg}$ \\
Vit. C & $19.50 \mathrm{mg}$ \\
\hline
\end{tabular}


índice de adequação (Tab. 3) verificouse um teor de adequação de quase $100 \%$ para proteínas (origem animal) e vitamina C, $82 \%$ de cálcio; $51 \%$ de vitamina $\mathrm{A}$ e $31 \%$ de ferro (origem animal), das recomendações diárias para pré-escolares.

Quanto aos escolares, a qualidade da merenda no que diz respeito a calorias e nutrientes, mostrou-se deficiente para calorias, em $65 \%$, vitamina A $76 \%$, Ferro $75 \%$, B1 $65 \%$, Niacina $60 \%$ e B2 $49 \%$ (Tab. 3).

$A$ análise do valor nutricional, leva-nos a deduzir que: a Merenda Escolar teve uma contribuição importante chegando muitas vezes a atender quase que integralmente as recomendações diárias em alguns nutrientes principalmente aos pré-escolares. Entretanto, vale salientar a necessidade de cardápios que contenham preparações com maior densidade energética, a partir de um aporte maior de lipídio na dieta, e a importância da utilização de hortaliças e frutos regionais em proporções adequadas e com mais freqüência por esses grupos populacionais. É uma forma de

Tabela 3. Contribuição percentual de nutrientes da merenda escolar de préescolares e escolares de Palmeira do Javari, em relação às recomendações (RDA).

\begin{tabular}{|c|c|c|}
\hline $\begin{array}{c}\text { Calorias } \\
\text { e } \\
\text { Nutrientes \% }\end{array}$ & $\begin{array}{l}\text { Grupos popt } \\
\text { Pré-escolares }\end{array}$ & $\begin{array}{l}\text { lacionais } \\
\text { Escolares }\end{array}$ \\
\hline $\begin{array}{l}\text { Calorias } \\
\text { Proteína } \\
\text { Cálcio } \\
\text { Ferro } \\
\text { Vit.A } \\
\text { Vit.B1 } \\
\text { Vit.B2 } \\
\text { Niacina } \\
\text { Vit.C }\end{array}$ & $\begin{array}{c}55 \\
98 \\
82 \\
31 \\
51 \\
60 \\
82 \\
63 \\
97.5\end{array}$ & $\begin{array}{c}35 \\
59 \\
62 \\
25 \\
24 \\
35 \\
51 \\
40 \\
84.5\end{array}$ \\
\hline
\end{tabular}

introduzir um novo hábito alimentar e de corrigir as deficiências vitamínicas e de minerais. Os resultados relativos à prevalência de anemia podem ser observados na Tabela 4 , onde $53,8 \%$ dos pré-escolares eram anêmicos e apresentavam uma anemia severa. Enquanto que $28,5 \%$ dos escolares apresentavam anemia severa e $25 \%$ anemia moderada (Tab. 4).

Verificou-se portanto que mais de $50 \%$ das crianças estudadas eram anêmicas, o que nos leva a situar a anemia como uma deficiência nutricional, fato já observado em outros estudos na região de Manaus (CONTENTE, 1963; GIUGLIANO et al., 1977; 1978; 1981). Um dos fatores que associamos ao elevado índice de anemia encontrado nesse estudo, foi a alta incidência de parasitose intestinal e uma limitada ingestão dietética que promoveria um efeito carencial acumulativo, a partir do baixo consumo de calorias e de ferro de boa qualidade. $O$ exame parasitológico dos 26 pré-escolares foram positivos, sendo que $80,8 \%$ (21) eram portadores de parasitoses múltiplas. Nos escolares $94,6 \%$ (53) foram positivos e $58,9 \%$ (33) albergavam dois ou mais parasitos, sobressaindo Ancylostomídeos e

Tabela 4. Prevalência (\%) de anemia em pré-escolares de Palmeira do Javari, Am.

\begin{tabular}{lll}
\hline Classificaçåo & \multicolumn{2}{c}{ Grupos populacionais } \\
Pré-escolares & Escolares \\
\hline $\begin{array}{l}\text { Anemia severa } \\
\mathrm{Hb}<9.5 \mathrm{~g} / \mathrm{dl}\end{array}$ & (2) $7.7 \%$ & (16) $28.6 \%$ \\
$\begin{array}{l}\text { Anemia } \\
\mathrm{Hb}<11 \mathrm{~g} / \mathrm{dl}\end{array}$ & (12) $46.1 \%$ & (14) $25 \%$ \\
$\begin{array}{l}\mathrm{Normal} \\
\mathrm{Hb}>11 \mathrm{~g} / \mathrm{dl}\end{array}$ & (12) $46.1 \%$ & (26) $46.4 \%$ \\
\hline
\end{tabular}


Ascaris lumbricoides em 48,8\%, $T$. trichiurus $29,2 \%$ do total da população estudada. Alguns autores têm comprovado a incidência de verminose e o elevado índice de anemia na região, conforme estudos já realizados (COSTA, 1947; OLIVEIRA 1959; MONTENEGRO et al., 1963; PINHEIRO et al., 1976; GIUGLIANO et al., 1977; MANTORIL et al., 1978).

\section{CONCLUSÕES E SUGESTÕES}

De acordo com a classificação de GOMEZ (1956); 11,5\% dos 26 préescolares apresentaram desnutrição de grau I; $41 \%$ e $9 \%$ dos escolares apresentaram respectivamente, desnutrição de grau I e grau II.

Em relação a alimentação oferecida pela Merenda Escolar de Palmeiras aos pré-escolares e escolares, foi inadequada quanto ao aporte calórico, vitamina $\mathrm{A}$, ferro, $\mathrm{B} 1, \mathrm{~B} 2$ e niacina. Sugere-se cardápios que contenham preparações com maior densidade energética a partir de um aporte maior de lipídio na dieta. Outro aspecto relevante é quanto a adequação de vitaminas e minerais, através de uma oferta maior de frutas e hortaliças regionais que são reduzidas.

A alta incidência de infestações parasitárias encontrada nesse estudo, pode ser um reflexo de um saneamento básico inadequado, favorecendo uma exposição maior a esses agentes expoliadores, podendo causar reflexos negativos ao estado nutricional. Isso nos leva a sugerir programas de melhoramento da rede de esgoto, distribuição de água tratada, programas de conscientização em relação ao saneamento básico e higiene.

É importante que, associado a uma alimentação equilibrada, haja um meio social, econômico e cultural adequado para o crescimento e desenvolvimento de uma criança, e de outros segmentos da sociedade.

\section{Referências bibliográficas}

AZOUBEL, L. M. O.; GARCIA, R. W. D.; NEVES, J. M. V. 1982. Tabela de Composição de Alimentos. In: DUTRA DE OLIVEIRA;J.E.; WILSON,E.D.; SANTOS, A.C. Nutrição básica, São Paulo, sarvier, p. 249-668.

CONTENTE, J. J. S. 1963. Estudo clíniconutricional em menores da cidade de Manaus. Rev. Ass. Med. Bras., 9:169-180.

COSTA, O. R. 1947. Incidência de parasitas intestinais em 4 cidades da Amazônia. Rev. Serv. Esp. Saúde Publ,: 203-219.

FAUST, E. C.; SAWITZ, W.; TOBIE, J.; ODON, V.; PERES, C.; LICICONE, D. R. 1939. Comparative efficiency of various technics for the diagnosis of protozoa and helmints in fezes. $J$. Parasit., 25:241-62.

FUNDAÇÃO INSTITUTO BRASILEIRO DE GEOGRAFIA E ESTATISTICA.(FIBGE). 1977. Tabela de composição de alimentos. Estudo Nacional de Defesa Familiar. Rio de Janeiro, V.3. publicações especiais.

GIUGLIANO, R.; SHRIMPTON, R. 1977. Estudo antropométrico e clinico do estado nutricional em um grupo de crianças pré-escolares de Manaus. Acta Amazonica, 7(3):389-394.

GIUGLIANO, R.; ALBUQUERQUE, H.C.R.; SHRIMPTON, R. 1978. Estudo antropométrico, clínico e de padrões alimentares em um grupo de Escolares de 
Manaus. Acta Amazonica, 8(1):75-82.

GIUGLIANO, R.; GIUGLIANO, L.; SHRIMPTON, R. 1981. Estudos nutricionais das populações rurais da Amazônia. I. Várzea do Rio Solimões. Acta Amazonica, 11(4):773-788.

GIUGLIANO, R.; SHRIMPTON, R.; MARINHO, H.A.; GIUGLIANO, L. 1984. Estudos nutricionais das populações rurais da Amazônia. II. Rio Negro. Acta Amazonica, 14(3-4):427449.

GOMEZ, F. 1956. Mortality in second and third degree. Malnutrition. J. Trop. Ped., 2:77-84

HARTMAN, A. F.; JORGE JOÃO, W, S. 1978. Desnutrição protéico-calórica na região centro-Amazônica. Relatório preliminar da prevalência e fatores demográficos. J. Ped, 45,:323-32.

JELLIFFE, D. B. 1966. The evaluation of the nutritional status of the community, Geneva. WHO Monograth N. 53.

MONTENEGRO, L.; BATISTA, D.; OLIVEIRA, W. R. 1963. Novas observações higiênicosociais no núcleo agrícola japonês da estrada Manaus-Itacoatiara, Estado do Amazonas. $O$. Hospital., 63:129-134.

MONTORIL, F. M.; FERRARONI, J. J.; MANTORIL, O. A. L. 1978. Diagnóstico sócio-parasito-sanitário da cidade de Nova Olinda do Norte/Amazonas. Acta Amazonica, 8(1):91-98.

NATIONAL ACADEMY OF SCIENCES, NATIONAL RESEARCH COUNCIL. 1980. Recommended dietary allowances. 9th ed. Washington. D.C.
NELSON, W. E. 1979. Textbook of Pediactrics. 11 ed. Philadelphia, W. B. Saunders Company. pg. 33-41.

OLIVEIRA, W. R. 1959. Contribuição ao estudo coprológico na cidade de Manaus. Brasil médico, 73(7/28):123-125.

ORNELLAS, L. H. 1983. Alimentação da criança, 2 ed. São Paulo, Atheneu.

OMS. 1968. Anemias nutricionales. Genebra. Informe de um grupo de experts, OMS. Genebra. (serv.inf. tecn., 405).

PINHEIRO, M. F. S.; VASCONCELOS, J. C.; WENDEL. D. E. 1976. Contribuição ao estudo de parasitas intestinais em dois bairros de Manaus, Amazonas. Acta Amazonica, 6:67-73.

SHRIMPTON, R.; GIUGLIANO, R. 1979. Consumo de alimentos e alguns nutrientes em Manaus, Amazonas. Acta Amazonica, 9:117-141.

SIMPÓSIO BRASILEIRO DE ALIMENTAÇÃO E NUTRIÇÃO 7. 1984. Relatório da região Norte, Niterói, Anais... Rio de Janeiro, Fundação, SIBAN, Interciência, p. 55-69.

VENTAKACHALAN, P. S. 1976. Nutritonal Nuances of the term pre-school child and the need, for precise definition: In: Perfil Estatístico de Crianças e Mães no Brasil. Aspectos Nutricionais. 1974 - 5, FIBGE. 